\title{
Preliminary screening of differentially expressed circulating microRNAs in patients with steroid-induced osteonecrosis of the femoral head
}

\author{
XINGSHAN WANG* ${ }^{*}$ WENWEI QIAN* ${ }^{*}$ ZHIHONG WU, YANYAN BIAN and XISHENG WENG \\ Department of Orthopaedic Surgery, Peking Union Medical College Hospital, \\ Peking Union Medical College \& Chinese Academy of Medical Sciences, Beijing 100730, P.R. China
}

Received December 1, 2013; Accepted August 7, 2014

DOI: $10.3892 / \mathrm{mmr} .2014 .2660$

\begin{abstract}
RNAs have been shown to be stable and detectable in circulating blood, and circulating microRNAs are specifically expressed in numerous diseases. However, to date, the association between microRNAs and osteonecrosis of the femoral head (ONFH) has remained elusive. It was hypothesized that specific microRNAs in the serum of ONFH patients may participate in the pathogenesis of ONFH. In the present study, therefore, the ONFH-specific serum microRNAs were screened using deep sequencing technology. Peripheral blood serum was collected from three steroid-induced ONFH patients with systemic lupus erythematosus (SLE), three SLE controls and three healthy controls, respectively. Total RNA was extracted from the serum and a microRNA cDNA library was built. High-throughput sequencing was then used to sequence the serum microRNAs of the samples and screen differentially expressed serum microRNAs in patients with steroid-induced ONFH. 27 differentially expressed microRNAs from ONFH serum were selected; of these, 15 microRNAs were over-expressed and 12 were under-expressed compared with SLE controls and healthy controls. The present study showed that abnormally expressed microRNAs existed in the serum of ONFH patients and therefore have the potential to be diagnostic markers.
\end{abstract}

Correspondence to: Dr Xisheng Weng, Department of Orthopaedic Surgery, Peking Union Medical College Hospital, Peking Union Medical College \& Chinese Academy of Medical Sciences, 1 Shuaifuyuan Wangfujing Street, Dongcheng District, Beijing 100730, P.R. China

E-mail: wengxsh@126.com

*Contributed equally

Key words: osteonecrosis of the femoral head, systemic lupus erythematosus, microRNA, sequencing

\section{Introduction}

A previous study indicated that osteonecrosis of the femoral head $(\mathrm{ONFH})$ may be associated with injury of endothelial cells, abnormal differentiation of stem cells, osteocyte apoptosis and hypercoagulation (1), while microRNAs were shown to participate in the regulation of these process $(2,3)$, suggesting that microRNAs may be involved in the onset and progression of ONFH. Therefore, circulating microRNAs provide a novel approach for the early diagnosis of the disease.

Different types of cells have characteristic microRNA expression profiles, and changes in expression are associated with physiological development (1) and disease progression (3). The different expressions of microRNA may not only be the foundation of its function, but also be used as diagnostic markers, which has been demonstrated in oncological studies (4-6).

Circulating microRNAs (miRs) are stable and detectable, and can be purified and quantified in serum samples. For instance, miR-221 is abnormally expressed in malignant melanoma (MM) cells. The serum levels of miR-221 were found to be significantly higher in patients with stage I-IV MM compared to those with MM in situ, and were correlated with tumor thickness (7). MicroRNA-21 levels were elevated in the serum of patients with breast cancer (8), and a study on microRNA in the serum of patients with lung cancer had similar results (9). These results indicated that microRNAs may be potential diagnostic markers of cancer. The role of circulating microRNAs in the diagnosis of other diseases has also been studied. Wang et al (10) found the serum levels of microRNA-208a increased in patients with acute myocardial infarction, and this change was specific. Similarly, another study found that miR423-5p was specifically enriched in the blood of patients with heart failure and further analysis demonstrated that miR423-5p was a diagnostic predictor of heart failure (11). Levels of vascular-specific microRNAs such as miR-126 were found to be decreased in serum of patients with stable coronary heart disease (12). In general, circulating microRNAs showed great potential as diagnostic markers for tumor and circulatory diseases. However, to the best of our knowledge, the use of circulating microRNA for diagnosis of ONFH has not yet been reported to date. 
In the present study, microRNAs were purified from the serum of patients with steroid-induced ONFH and controls. High-throughput sequencing was then performed to screen the differentially expressed microRNAs from the serum of patients with steroid-induced ONFH.

\section{Materials and methods}

Serum collection. Blood samples were obtained from three patients with ONFH secondary to hormone application for the treatment of systemic lupus erythematosus (SLE), three matched controls with SLE and similar hormone application history but no ONFH, and three healthy controls. The present study was approved by the ethics committee of Peking Union Medical College Hospital (Beijing, China). All donors provided written informed consent and the local institutional review board approved the study. Serum was separated from the sample within $1 \mathrm{~h}$ after blood was derived and then stored at $-80^{\circ} \mathrm{C}$.

Extraction of RNA. An MiRNeasy Mini kit (217004; Qiagen, Hilden, Germany) was used to extract the total RNA from the serum. Total RNA was dissolved in $300 \mu$ l elution buffer (19086; Qiagen), and checked by a Nanodrop 2000 (Thermo Fisher Scientific, Waltham, MA, USA). The total RNA was concentrated following centrifugation.

Extraction of microRNA. For preparation of the Tris-borate EDTA (TBE)-Urea PAGE run (Invitrogen Life Technologies, Carlsbad, CA, USA), $2 \mu \mathrm{l}$ SRA ladder (Illumina, Inc., San Diego, CA, USA) was mixed with $2 \mu \mathrm{l}$ SRA gel loading dye (Illumin, Inc.) in a sterile, RNase-free 200- $\mu$ l PCR tube (Extra Gene, Inc., Taichung City, Taiwan). $10 \mu$ l total RNA was mixed with $10 \mu$ l SRA gel loading dye in a sterile RNase-free 200- $\mu$ l PCR tube. The sample and ladder tubes were heated at $65^{\circ} \mathrm{C}$ for $5 \mathrm{~min}$ on a thermal cycler (S1000; Bio-Rad Laboratories, Inc., Hercules, CA, USA) and then placed on ice. The samples were centrifuged (5417R; Eppenderf, Hamburg, Germany) at $18,000 \mathrm{x} \mathrm{g}$ for $10 \mathrm{sec}$ at room temperature to collect the entire column of the tube. Both the entire SRA ladder and the sample RNA were loaded on the same gel with several lanes between them. The gel was run at $200 \mathrm{~V}$ for $1 \mathrm{~h}$. The bottom of a sterile, nuclease-free, 0.5-ml microcentrifuge tube (Extra Gene, Inc.) was punctured 4-5 times with a 21-gauge needle (Sigma-Aldrich, St. Louis, MO, USA). The $0.5-\mathrm{ml}$ microcentrifuge tube was placed into a sterile, round-bottom, nuclease-free, 2-ml microcentrifuge tube (Extra Gene, Inc.). The cassette was pried apart and the gel was stained with TBE/ethidium bromide (Life Technologies, Waltham, MA, USA) in a clean container for $2 \mathrm{~min}$. The gel was viewed on a Dark Reader transilluminator (Clare Chemical Research, Dolores, CO, USA). The SRA ladder ranged from 20-100 bases in 10-base increments. Using a clean scalpel (Swann-Morton, Sheffield, UK), a band of the gel corresponding to the 18-30 nucleotide bands in the marker lane were excised and placed into the $0.5-\mathrm{ml}$ microcentrifuge tube punctured with a 21-gauge needle. The stacked tubes were centrifuged on a benchtop microcentrifuge at 20,000 $\mathrm{x} g$ for $2 \mathrm{~min}$ at room temperature to push the gel through the holes into the 2-ml tube. $300 \mu 10.3 \mathrm{M} \mathrm{NaCl}$ (Illumina, Inc.) was added to the SRA gel debris in the 2-ml tube and the RNA was eluted by rotating the tube gently at room temperature for $4 \mathrm{~h}$. The eluate and the gel debris were transferred to the top of a Spin-X cellulose acetate filter (Illumina, Inc.). The filter was then centrifuged on a benchtop microcentrifuge at 20,000 $\mathrm{x} \mathrm{g}$ for $2 \mathrm{~min}$ at room temperature. $1 \mu \mathrm{l}$ glycogen in solution $(10 \mathrm{mg} / \mathrm{ml}$; Illumina, Inc.) and $750 \mu 1100 \%$ ethanol (Sigma-Aldrich) were added to the Spin-X tube at room temperature followed by incubation at $-80^{\circ} \mathrm{C}$ for $30 \mathrm{~min}$. The Spin-X tube was then centrifuged on a microcentrifuge at $20,000 \mathrm{x} \mathrm{g}$ and $4^{\circ} \mathrm{C}$ for $25 \mathrm{~min}$. The supernatant was discarded and the pellet washed with $750 \mu \mathrm{l}$ $75 \%$ ethanol (Sigma-Aldrich) at room temperature. The supernatant was discarded and the pellet was air-dried. The RNA pellet was resuspended in $5.7 \mu$ l ultra pure water (Illumina, Inc.).

Ligation of the 5' and 3' adapter. The microRNA was transferred into a 200- $\mu$ l PCR tube. $1.3 \mu \mathrm{l} \mathrm{SRA} \mathrm{5'}$ adapter, $1 \mu 1$ 10X T4 RNA ligase buffer, $1 \mu \mathrm{l}$ of RNase inhibitor and $1 \mu \mathrm{l}$ T4 RNA ligase, all purchased from Illumina, Inc., were added to each tube of PAGE gel-isolated microRNA. The starting volume of microRNA was $5.7 \mu 1$. Samples were incubated at $20^{\circ} \mathrm{C}$ for $6 \mathrm{~h}$ on a thermal cycler and kept at $2-8^{\circ} \mathrm{C}$ overnight. The reaction was terminated by adding $10 \mu \mathrm{l}$ SRA gel loading dye. The $15 \%$ TBE-urea gel was run, the band of the gel corresponding to the 40-60 nucleotide bands in the marker lane was excised, and the sample RNA was isolated according to the aforementioned method. The RNA pellet was resuspended in $6.4 \mu \mathrm{l}$ ultra pure water.

The small RNA was transferred into a $200-\mu 1$ PCR tube. $0.6 \mu 1$ SRA 3' adapter (Illumina, Inc.), $1 \mu 1$ 10xT4 RNA ligase buffer, $1 \mu 1$ RNase inhibitorand, $1 \mu 1$ T4 RNA ligase were added to each tube of the PAGE gel-isolated microRNA. The starting volume of the microRNA was $6.4 \mu \mathrm{l}$. Samples were incubated at $20^{\circ} \mathrm{C}$ for $6 \mathrm{~h}$ on a thermal cycler and held overnight at $2-8^{\circ} \mathrm{C}$. The reaction was terminated by adding $10 \mu \mathrm{l}$ SRA gel loading dye. The $15 \%$ TBE-urea gel was run, the band of the gel corresponding to the 70-90 nucleotide bands in the marker lane was excised, and the sample RNA was isolated according to the previous method. The RNA pellet was resuspended in $4.5 \mu \mathrm{l}$ ultra pure water.

Reverse transcription $(R T)$ and polymerase chain reaction (PCR) amplification. $5 \mu 1$ purified 5' and 3' ligated RNA and $0.5 \mu \mathrm{l}$ SRA RT primer (Illumina, Inc.) were combined in a sterile RNase-free $200-\mu 1$ microcentrifuge tube. The mixture was heated at $65^{\circ} \mathrm{C}$ on a thermal cycler for $10 \mathrm{~min}$ and the tube was placed on ice. $2 \mu 15 \mathrm{X}$ First Strand buffer (Illumina, Inc.), $0.5 \mu 112.5 \mathrm{mM}$ dNTP Mix (Illumina, Inc.), $1.0 \mu 1100 \mathrm{mM}$ dithiothreitol (Promega, Maddison, WI, USA) and $0.5 \mu 1$ RNase inhibitor were added to the sample. The sample was heated on the thermal cycler to $48^{\circ} \mathrm{C}$ for $3 \mathrm{~min}$, and $1 \mu \mathrm{l}$ SuperScript II Reverse Transcriptase (Invitrogen Life Technologies) was then added. The total volume was now $10 \mu \mathrm{l}$. The sample was incubated on the thermal cycler at $44^{\circ} \mathrm{C}$ for $1 \mathrm{~h}$.

$40 \mu$ PCR master mix (Illumina, Inc.) was added into a sterile, nuclease-free 200- $\mu$ 1 PCR tube. $10 \mu 1$ single strand reverse-transcribed cDNA was added. The PCR amplification was run on a thermal cycler using the following PCR conditions: $30 \mathrm{sec}$ at $98^{\circ} \mathrm{C} ; 10 \mathrm{sec}$ at $98^{\circ} \mathrm{C}, 30 \mathrm{sec}$ at $60^{\circ} \mathrm{C}, 15 \mathrm{sec}$ at $72^{\circ} \mathrm{C}$, and repeat for 15 cycles; $10 \mathrm{~min}$ at $72^{\circ} \mathrm{C}$; Hold at $4^{\circ} \mathrm{C}$. 

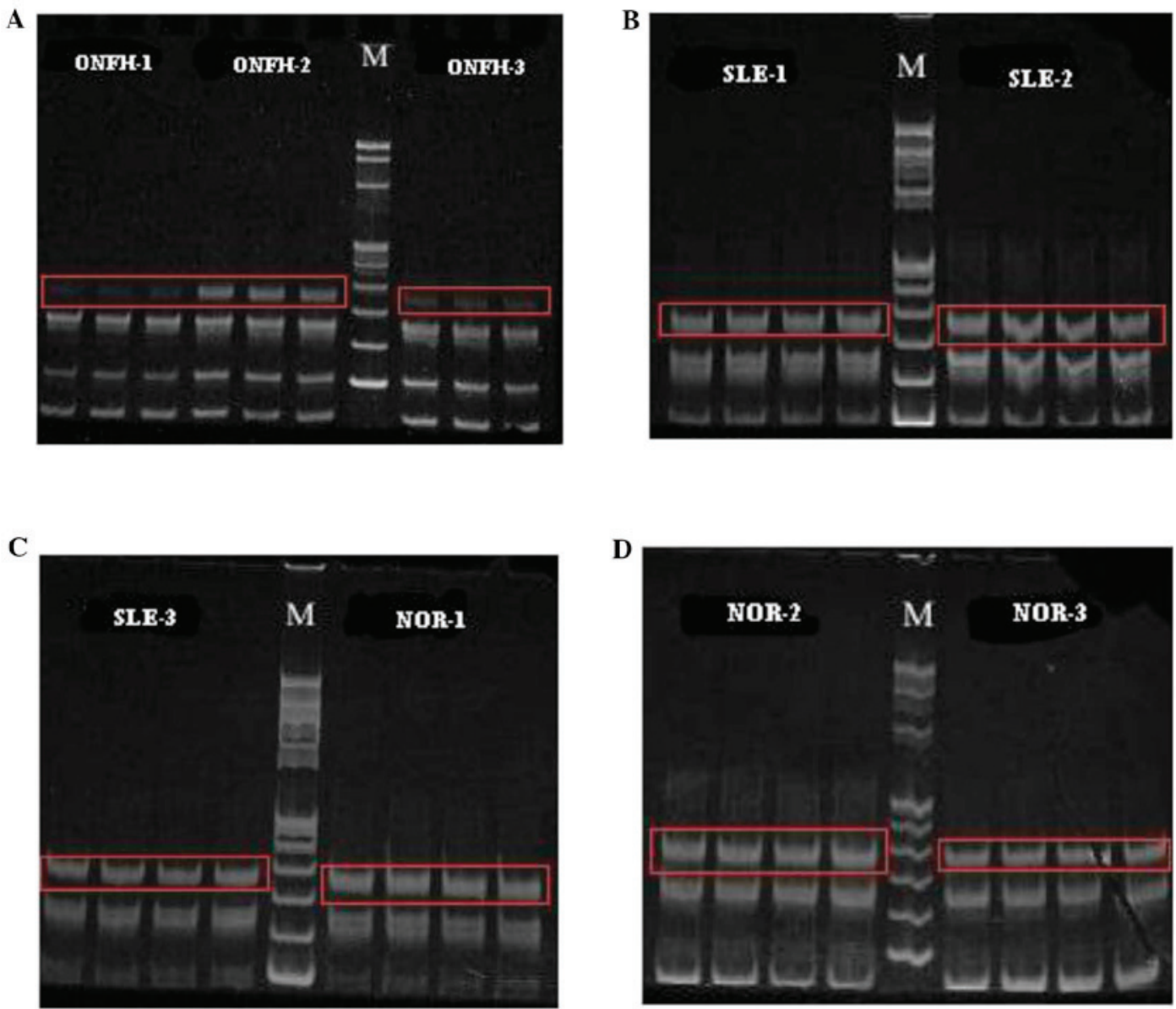

Figure 1. Isolation of cDNA obtained by reverse transcription polymerase chain reaction of microRNA from blood samples of patients by gel electrophoresis. (A) Three samples of patients with ONFH are represented as ONFH-1, ONFH-2 and ONFH-3, respectively. (B and C) Three samples of SLE controls are represented as SLE-1, SLE-2 and SLE-3, respectively. (C and D) Three samples of healthy controls are represented as NOR-1, NOR-2 and NOR-3, respectively. The brightest band shown in each image corresponds to the 100-bp band. The red zones indicate the bands corresponding to the 140-nucleotide bands, which were excised and used for RNA isolation and screening. M, marker; ONFH, osteonecrosis of the femoral head; SLE, systemic lupus erythematosus; NOR, control.

Purification and test of the cDNA profile. Two lanes of a 6\% PAGE gel (Invitrogen Life Technologies) were loaded with $25 \mu \mathrm{l}$ of mixed amplified cDNA construct each and loading dye. The gel was run for 30-35 min at $200 \mathrm{~V}$. A 140-bp band was excised from the sample lanes and the sample cDNA was isolated according to the aforementioned method. The pellet was resuspended in $10 \mu 1$ resuspension buffer (Illumina, Inc.).

The product was tested by using the KAPA Library Quantification kit (KAPA Biosystems, Woburn, MA, USA). Eligibility criteria for quantitative PCR were that the concentration was $>1 \mathrm{nM}$ and that no dimer pollution was present.

High-throughput sequencing. The purified DNA was sequenced by an Illumina HiSeq 2000 Sequencer (Illumina, Inc.) according to the manufacturer's instructions. Then the raw data generated by the sequencer were processed to produce digital-quality data. The sequencing quality was evaluated, and the length distribution of the microRNA reads was calculated. Then adapter, low quality reads, reads $<18 \mathrm{nt}$ as well as reads for non-coding, ribosomal, transfer, messenger, small nuclear and small nucleolar RNAs were eliminated. Finally, clean reads were compared with miRBase 18.0 , as previously described (13) and the total copy number of each sample was normalized to 100,000 .
Statistical analysis. The samples were divided into three groups. Each group contained one ONFH patient, one matched SLE control and one healthy control. A chi-squared test was performed to compare the microRNA expression difference of the ONFH patient with that of the SLE control and healthy control, respectively, in each group. SPSS 16.0 software (SPSS, Inc., Chicago, IL, USA) was used for statistical analyses. $\mathrm{P}<0.05$ was considered to indicate a statistically significant difference between values. The candidate microRNAs were screened out when they were differentially expressed in all of the three groups and the trend of the expression was consistent.

\section{Results}

Profiles of microRNA cDNA. Following reverse transcription, a band of gel corresponding to the 140 nucleotide bands in the marker lane was excised and used for isolation of the sample cDNA (Fig. 1).

The profiles were evaluated by quantitative PCR and the results demonstrated that the profiles were eligible for high-throughput sequencing (Table I).

Results of high-throughput sequencing. Raw data were obtained following sequencing. Clean reads were gained 
Table I. microRNA concentration in samples.

\begin{tabular}{lcc}
\hline Samples $(\mathrm{n}=9)$ & Concentration $(\mathrm{nM})$ & Twin peaks \\
\hline ONFH-1 & 15.33 & No \\
ONFH-2 & 40.53 & No \\
ONFH-3 & 7.22 & No \\
SLE-1 & 44.52 & No \\
SLE-2 & 53.02 & No \\
SLE-3 & 32.22 & No \\
NOR-1 & 70.81 & No \\
NOR-2 & 39.72 & No \\
NOR-3 & 62.02 & No \\
\hline
\end{tabular}

In all cases, the concentration was $>1 \mathrm{nM}$. All of the profiles were eligible for high-throughput sequencing. ONFH, osteonecrosis of the femoral head; SLE, systemic lupus erythematosus; NOR, control.

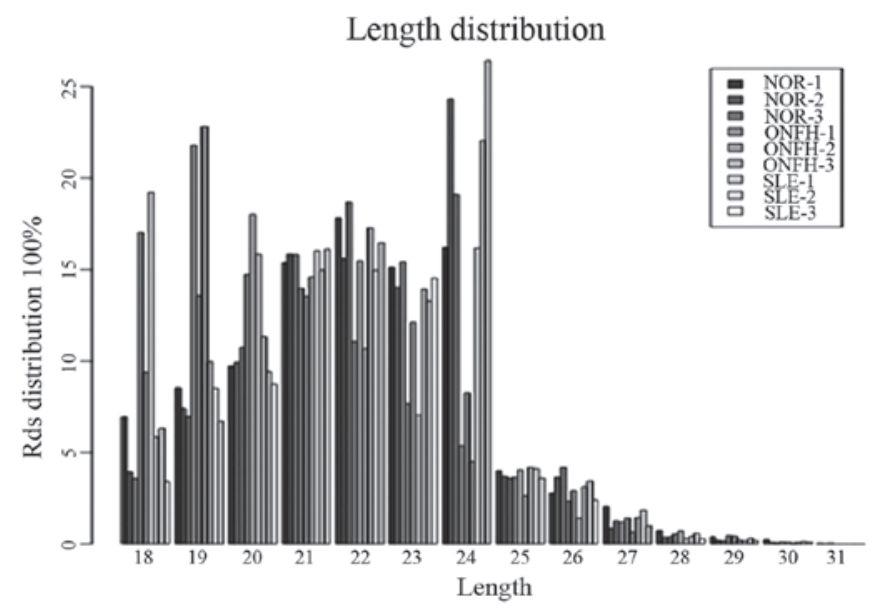

Figure 2. The proportion of $18-20$-bp clean reads was higher in the $\mathrm{ONFH}$ group than that in the other two groups, while the proportion of 22-24-bp clean reads was lower in the ONFH group than that in the other two groups, suggesting ONFH contained different microRNA clean reads compared with the other two groups. ONFH, osteonecrosis of the femoral head; SLE, systemic lupus erythematosus; NOR, control.

following subsequent analysis. From these data, a length distribution was generated according to the distribution of the clean reads (Fig. 2).

Twenty seven candidate microRNAs were screened out from ONFH serum. Fifteen microRNAs, including miR-423-5p, miR-3960, miR-195-5p, miR-15b-3p and miR-1304-3p, were over-expressed. Twelve microRNAs, including miR-100-5p, miR-99a-5p, miR-532-5p, miR-140-5p, miR-10a-5p, miR-10b-5p, miR-181c-5p and miR-433, were under-expressed in ONFH serum compared with SLE controls and healthy controls (Fig. 3).

\section{Discussion}

Lawrie et al (14) firstly described circulating microRNAs and found that microRNAs may have potential as non-invasive diagnostic markers. Another study reported that serum microRNAs were resistant to RNase A digestion and remained stable when serum was treated for $3 \mathrm{~h}$ at low (1) or high (13) $\mathrm{pH}$. Expression levels of serum microRNAs were reproducible and consistent among individuals (15). This feature has laid the foundation for using circulating microRNAs in disease diagnosis.

In the last five years, most of the studies on circulating microRNA were focused in the field of oncology (16-18). MicroRNAs-21, 92, 93, 126 and 29a were significantly over-expressed in the serum of cancer patients compared to those of controls (19). Gradual elevation of circulating let-7a, let-7f, miR-34a, miR-98, miR-331, miR-338 and miR-652 with progression of hepatocarcinogenesis was demonstrated (20). Levels of let-7a, let-7f and miR-98 significantly increased in the serum of rats even at very early stages according to the RT quantitative PCR analysis (20). This showed the possibility of serum microRNAs being used for the diagnosis and monitoring of the progress of tumor development.

However, to the best of our knowledge, the correlation of circulating microRNAs with ONFH has not been reported.

The present study first measured the serum levels of microRNAs in patients with steroid-induced ONFH. Levels of 27 microRNAs were found to be significantly changed in steroid-induced ONFH patients. Several of these microRNAs were involved in the regulation of apoptosis, cell proliferation and differentiation of stem cells in accordance with other previous studies.

Apoptosis-associated microRNAs. miR-195-5p and miR-15b-3p are members of the miR-15/16 family, while the miR-15/16 family has an important regulatory role in apoptosis. Xia et al (21) found that overexpression of miR-15b or miR-16 sensitized multidrug-resistant gastric cancer SGC7901/VCR cells to vincristine-induced apoptosis and reduced B-cell lymphoma $2(\mathrm{Bcl}-2)$ protein levels. This study suggested that miR-15b and miR-16 may be involved in the modulation of apoptosis via targeting $\mathrm{Bcl}-2$. Another study found that upregulation of miR-15 family members may participate in hypoxia-induced cardiomyocyte cell death, and that underexpression of miR15 family members reduced hypoxia-induced myocardial cell death (22). By comparing levels of miR-195 in colorectal cancer tissues and matched non-neoplastic mucosa tissues, Liu et al (23) found that miR-195 was downregulated in cancer tissues. Anti-apoptotic Bcl-2 was directly targeted by miR-195, and restoration of miR-195 could promote cell apoptosis and suppress tumorigenicity (23).

In this study, serous levels of miR15b-3p and miR195-3p were upregulated in patients with steroid-induced ONFH, compared with those in the control group. The apoptosis-promoting effect of miR15 and the miR195 may be involved in the process of femoral head necrosis; however, this hypothesis remains to be confirmed by further experimental studies.

Cell proliferation-associated microRNAs. The miR-10 family was reported to participate in regulating the proliferation of neuroblastoma cells (24) and smooth muscle cells (25), and promoting the differentiation of endoderm cells into liver cells (26). In addition, miR-10a was shown to be involved in the regulation of kidney development alongside miR-106b and 


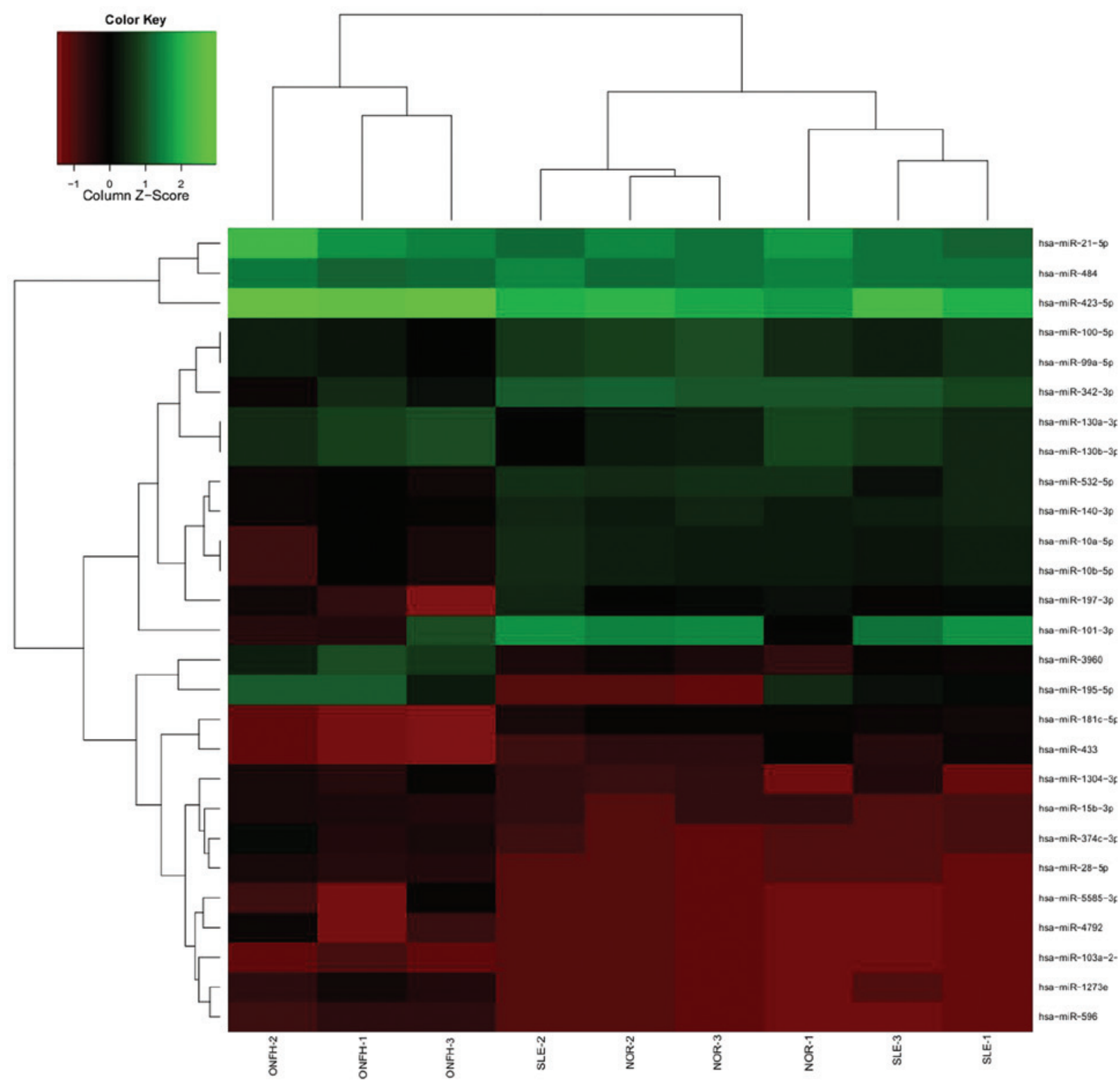

Figure 3. Heat map was drawn according to statistical analysis. Green color represents up-regulation of microRNAs and red color represents down-regulation of microRNAs. The expression of microRNAs in ONFH serum was different from that in the controls. ONFH, osteonecrosis of the femoral head; SLE, systemic lupus erythematosus; NOR, control; miR, microRNA; hsa, Homo sapiens.

miR-17-5p. The absence of these microRNAs caused kidney failure prior to maturity, concurrent with cell apoptosis and increased expression of pro-apoptotic protein (27). In pancreatic cancer cells, miR-10a was also highly expressed, and may participate in the invasion of pancreatic cancer by inhibiting homeobox A (Hoxa)1 (28). The upregulation of miR-10b promoted the migration of microvascular endothelial cells (HMEC-1) and angiogenesis (29). miR-10b was also strongly upregulated in low-grade and high-grade gliomas, and inhibition of miR-10b reduced glioma cell growth by cell-cycle arrest and apoptosis (30).

All these studies indicated the role of miR-10a and miR-10b as positive regulators of cell proliferation and differentiation. Meanwhile, in the present study, downregulation of miR-10a and miR-10b was detected in the serum of ONFH patients, which may inhibit cell proliferation, differentiation and angiogenesis. This may finally lead to the occurrence of ONFH.
Mesenchymal stem cell differentiation-associated microRNAs. Transforming growth factor- $\beta$ (TGF- $\beta$ ) has a crucial role in tissue regeneration, cell differentiation and embryonic development (31). TGF- $\beta$ inhibited the expression of the core-binding factor alpha 1 (Cbfa1) and osteocalcin genes, which was mediated by Smad3. This repression occurred in mesenchymal cells and provided a central regulatory mechanism for the inhibition of osteoblast differentiation by TGF- $\beta$ (32). In addition, Runtrelated transcription factor 2 (Runx2)/Cbfa1/acute myeloid leukemia 3 are important transcription factors which regulate osteoblast differentiation (33-35) and cell function (36). Mice with knockout of Runx2 lacked osteoblasts and presented with bone development disorders $(33,34)$. Hjelmeland et al (37) found that dermal fibroblasts acquired an osteoblast-like phenotype in the absence of Smad3 and that negative regulation of Runx2 activity by Smad3 in dermal fibroblasts prevented differentiation of mesenchymal lineages toward the osteoblastic lineage (37). 
Therefore, Smad3 is a key protein, which regulates osteogenic differentiation of mesenchymal stem cells. Pais et al (38) identified Smad3 as a direct miR-140 target and miR-140 suppressed the TGF $\beta$ and Runx 2 pathway through targeting Smad3, which resulted in promoted osteogenic differentiation of mesenchymal stem cells (38). In the present study, the expression of miR-140 was downregulated in patients with osteonecrosis of the femoral head. Decreased miR-140 may inhibit the differentiation of mesenchymal stem cells into bone and therefore participate in the occurrence of osteonecrosis.

Another noteworthy finding of the present study was that the serous levels of miR-3960 in ONFH patients increased significantly. Another recent study showed that miR-3960 targeted at Hoxa2, a repressor of Runx 2 expression, and regulated osteoblast differentiation through a regulatory feedback loop with Runx2, miR-3960 and miR-2861. Overexpression of miR-3960 promoted bone morphogenetic protein 2 -induced osteoblastogenesis, while the inhibition of miR-3960 expression attenuated the osteoblastogenesis (39). In the present study, the expression of miR-3960 in ONFH patients increased significantly, which suggested there may be a compensatory mechanism to promote osteoblast differentiation, while osteogenic differentiation of mesenchymal stem cells was inhibited in ONFH patients. However, this likely compensation may fail to reverse the process of osteonecrosis of the femoral head. Certainly this hypothesis still requires to be tested.

In conclusion, the present study showed that abnormally expressed microRNAs existed in the serum of patients with ONFH. Through further research, characteristic microRNAs were identified as potential markers for the early diagnosis of ONFH. The dysregulation of microRNAs may inhibit cell proliferation, osteoblast differentiation and promote apoptosis, but its exact role in the pathogenesis of ONFH remains to be elucidated. The role of microRNAs will be further clarified by ongoing and future studies.

\section{Acknowledgements}

This study was supported by grants from the National Natural Science Foundation of China (no. 81171775).

\section{References}

1. Kerachian MA, Séguin C and Harvey EJ: Glucocorticoids in osteonecrosis of the femoral head: a new understanding of the mechanisms of action. J Steroid Biochem Mol Biol 114: 121-128, 2009.

2. Miska EA, Alvarez-Saavedra E, Townsend M, et al: Microarray analysis of microRNA expression in the developing mammalian brain. Genome Biol 5: R68, 2004.

3. Lu J, Getz G, Miska EA, et al. MicroRNA expression profiles classify human cancers. Nature 435: 834-838, 2005.

4. Bloomston M, Frankel WL, Petrocca F, et al: MicroRNA expression patterns to differentiate pancreatic adenocarcinoma from normal pancreas and chronic pancreatitis. JAMA 297: 1901-1908, 2007.

5. Yanaihara N, Caplen N, Bowman E, et al: Unique microRNA molecular profiles in lung cancer diagnosis and prognosis. Cancer Cell 9: 189-198, 2006.

6. Schetter AJ, Leung SY, Sohn JJ, et al: MicroRNA expression profiles associated with prognosis and therapeutic outcome in colon adenocarcinoma. JAMA 299: 425-436, 2008.

7. Kanemaru H, Fukushima S, Yamashita J, et al: The circulating microRNA-221 level in patients with malignant melanoma as a new tumor marker. J Dermatol Sci 61: 187-193, 2011.
8. Asaga S, Kuo C, Nguyen T, Terpenning M, Giuliano AE and Hoon DS: Direct serum assay for microRNA-21 concentrations in early and advanced breast cancer. Clin Chem 57: 84-91, 2011.

9. Boeri M, Verri C, Conte D, et al: MicroRNA signatures in tissues and plasma predict development and prognosis of computed tomography detected lung cancer. Proc Natl Acad Sci USA 108: 3713-3718, 2011.

10. Wang GK, Zhu JQ, Zhang JT, et al: Circulating microRNA: a novel potential biomarker for early diagnosis of acute myocardial infarction in humans. Eur Heart J 31: 659-666, 2010.

11. Tijsen AJ, Creemers EE, Moerland PD, et al: MiR423-5p as a circulating biomarker for heart failure. Circ Res 106: 1035-1039, 2010.

12. Fichtlscherer S, De Rosa S, Fox H, et al: Circulating microRNAs in patients with coronary artery disease. Circ Res 107: 677-684, 2010.

13. Kozomara A and Griffiths-Jones S: miRBase: annotating high confidence microRNAs using deep sequencing data. Nucleic Acids Res 42: D68-D73, 2014

14. Lawrie CH, Gal S, Dunlop HM, et al: Detection of elevated levels of tumor-associated microRNAs in serum of patients with diffuse large B cell lymphoma. Br J Haematol 141: 672-675, 2008.

15. Chen X, Ba Y, Ma L, et al. Characterization of microRNAs in serum: a novel class of biomarkers for diagnosis of cancer and other diseases. Cell Res 18: 997-1006, 2008.

16. Syring I, Bartels J, Holdenrieder S, et al: Circulating serum microRNA (miR-367-3p, miR-371a-3p, miR-372-3p, miR-373-3p) as biomarkers for patients with testicular germ cell cancers. J Urol: Jul 18, 2014 (Epub ahead of print).

17. Slater EP, Strauch K, Rospleszcz S, et al: MicroRNA-196a and $-196 \mathrm{~b}$ as potential biomarkers for the early detection of familial pancreatic cancer. Transl Oncol 7: 464-471, 2014.

18. Sochor M, Basova P, Pesta M, et al: Oncogenic microRNAs: miR-155, miR-19a, miR-181b, and miR-24 enable monitoring of early breast cancer in serum. BMC Cancer 14: 448, 2014.

19. Resnick KE, Alder H, Hagan JP, et al: The detection of differentially expressed microRNAs from the serum of ovarian cancer patients using a novel real-time PCR platform. Gynecol Oncol 112: 55-59, 2009.

20. Sukata T, Sumida K, Kushida M, et al: Circulating microRNAs, possible indicators of progress of rat hepatocarcinogenesis from early stages. Toxicol Lett 200: 46-52, 2011.

21. Xia L, Zhang D, Du R, Pan Y, Zhao L, Sun S, et al: miR-15b and miR-16 modulate multidrug resistance by targeting BCL2 in human gastric cancer cells. Int J Cancer 123: 372-379, 2008.

22. Hullinger TG, Montgomery RL, Seto AG, Dickinson BA, Semus HM, Lynch JM, et al: Inhibition of miR-15 protects against cardiac ischemic injury. Circ Res 110: 71-81, 2012.

23. Liu L, Chen L, Xu Y, Li R and Du X: microRNA-195 promotes apoptosis and suppresses tumorigenicity of human colorectal cancer cells. Biochem Biophys Res Commun 400: 236-240, 2010.

24. Meseguer S, Mudduluru G, Escamilla JM, Allgayer H, and Barettino D: Micro-RNAs-10a and-10b contribute to retinoic acid-induced differentiation of neuroblastoma cells and target the alternative splicing regulatory factor SFRS1 (SF2/ASF) J Biol Chem 286: 4150-4164, 2011.

25. Huang H, Xie C, Sun X, Ritchie RP, Zhang J and Chen YE: miR-10a contributes to retinoid acid-induced smooth muscle cell differentiation. J Biol Chem 285: 9383-9389, 2010.

26. Kim N, Kim H, Jung I, Kim Y, Kim D and Han YM: Expression profiles of microRNAs in human embryonic stem cells during hepatocyte differentiation. Hepatol Res 41: 170-183, 2011.

27. Ho J, Pandey P, Schatton T, Sims-Lucas S, Khalid M, Frank MH, et al: The pro-apoptotic protein Bim is a microRNA target in kidney progenitors. J Am Soc Nephrol 22: 1053-1063, 2011.

28. Ohuchida K, Mizumoto K, Lin C, Yamaguchi H, Ohtsuka T, Sato N, et al: MicroRNA-10a is overexpressed in human pancreatic cancer and involved in its invasiveness partially via suppression of the HOXA1 gene. Ann Surg Oncol 19: 2394-2402, 2012.

29. Shen X, Fang J, Lv X, Pei Z, Wang Y, Jiang S and Ding K: Heparin impairs angiogenesis through inhibition of microRNA-10b. J Biol Chem 286: 26616-26627, 2011.

30. Gabriely G, Yi M, Narayan RS, Niers JM, Wurdinger T, Imitola J, et al: Human glioma growth is controlled by microRNA-10b. Cancer Res 71: 3563-3572, 2011.

31. Rosa A, Papaioannou MD, Krzyspiak JE and Brivanlou AH: miR-373 is regulated by TGF $\beta$ signaling and promotes mesendoderm differentiation in human Embryonic Stem Cells. Dev Biol 391: 81-88, 2014.

32. Alliston T, Choi L, Ducy P, et al: TGF-beta induced repression of CBFA1 by Smad3 decreases cbfal and osteocalcin expression and inhibits osteoblast differentiation (J). EMBO J 20: 2254-2272, 2001. 
33. Ducy P, Zhang R, Geoffroy V, Ridall AL and Karsenty G: Osf 2/Cbfa1: a transcriptional activator of osteoblast differentiation. Cell 89: 747-754, 1997.

34. Komori T, Yagi H, Nomura S, Yamaguchi A, Sasaki K, Deguchi K, Shimizu Y, Bronson RT, Gao YH, Inada M, Sato M, Okamoto R, Kitamura Y, Yoshiki S and Kishimoto T: Targeted disruption of Cbfa1 results in a complete lack of bone formation owing to maturational arrest of osteoblasts. Cell 89: 755-764, 1997.

35. Otto F, Thornell AP, Crompton T, Denzel A, Gilmour KC, Rosewell IR, Stamp GW, Beddington RS, Mundlos S, Olsen BR, Selby PB and Owen MJ: Cbfa1, a candidate gene for cleidocranial dysplasia syndrome, is essential for osteoblast differentiation and bone development. Cell 89: 765-771, 1997.
36. Ducy P, Starbuck M, Priemel M, Shen J, Pinero G, Geoffroy V, Amling M and Karsenty G: A Cbfa1-dependent genetic pathway controls bone formation beyond embryonic development. Genes Dev 13: 1025-1036, 1999.

37. Hjelmeland AB, Schilling SH, Guo X, et al: Loss of Smad3 mediated negative regulation of Runx 2 activity leads to an alteration in cell fate determination. Mol Cell Biol 25: 9460-9468, 2005.

38. Pais H, Nicolas FE, Soond SM, Swingler TE, Clark IM, Chantry A, et al: Analyzing mRNA expression identifies Smad3 as a microRNA-140 target regulated only at protein level. RNA 16: 489-494, 2010.

39. Hu R, Liu W, Li H, Yang L, Chen C, Xia ZY, et al: A Runx2/miR-3960/miR-2861 regulatory feedback loop during mouse osteoblast differentiation. J Biol Chem 286: 12328-12339, 2011. 